\title{
NR3C2 suppresses the proliferation, migration, invasion and angiogenesis of colon cancer cells by inhibiting the AKT/ERK signaling pathway
}

\author{
$\mathrm{JIA} \mathrm{LI}^{1,2}$ and $\mathrm{ZHAO} \mathrm{XU}^{2,3}$ \\ ${ }^{1}$ Nanchang University Jiangxi Medical College and ${ }^{2}$ Key Laboratory of Reproductive Physiology and \\ Pathology of Jiangxi Province, Nanchang University, Nanchang, Jiangxi 330031; \\ ${ }^{3}$ Queen Mary School, Nanchang University, Nanchang, Jiangxi 330027, P.R. China
}

Received July 26, 2021; Accepted September 23, 2021

DOI: $10.3892 / \mathrm{mmr} .2022 .12649$

\begin{abstract}
Nuclear receptor subfamily 3, group C, member 2 (NR3C2) serves an antitumorigenic role in several types of cancer; however, its role and mechanisms of action in colon cancer remains to be elucidated. The aim of the present study was to explore the effects of NR3C2 on the proliferation, migration, invasion and angiogenesis of colon cancer cells. The expression levels of NR3C2 in human colon epithelial NCM460 cells (spontaneously immortalized cell line) and colon cancer cell lines was detected using reverse transcription-quantitative PCR and western blotting. Cell Counting Kit-8 (CCK-8) and colony formation assays were used to assess cell viability and wound healing and Transwell assays were used to detect cell invasion and migration. ELISA was used to detect the expression levels of VEGF and tube formation assays were used to assess angiogenesis. The expression levels of angiogenesis-related proteins and AKT/ERK signaling pathway-related proteins were detected by western blotting. NR3C2 expression was downregulated in colon cancer cells and overexpression of NR3C2 inhibited proliferation, colony formation, migration and invasion of colon cancer cells. Overexpression of NR3C2 inhibited angiogenesis and activity of the AKT/ERK signaling pathway in colon cancer cells. Thus, it was demonstrated that NR3C2 inhibited the proliferation, colony formation, migration, invasion and angiogenesis of colon cancer cells through the AKT/ERK signaling pathway. These results may highlight novel targets for the treatment of colon cancer.
\end{abstract}

Correspondence to: Dr Zhao Xu, Queen Mary School, Nanchang University, 1299 Xuefu Avenue, Honggutan New District, Honggutan, Nanchang, Jiangxi 330027, P.R. China

E-mail: zhaoxuxu0791@163.com

Key words: NR3C2, colon cancer, AKT/ERK signaling pathway, angiogenesis, invasion

\section{Introduction}

Colon cancer is a common malignant tumor of the digestive system $(1,2)$. Colon cancer has high morbidity and mortality rates in developed western countries and is the third commonest tumor in the world (3-5). However, due to the rapid development of China's economy and improvements to the living standard of its citizens, the increases in life pressures and irregular work and rest patterns of the evolving population, the incidence and mortality of colon cancer in China are increasing. According to the latest data released by the National Cancer Center, there were 387,600 new colorectal cancer (CRC) cases in China in 2015 , accounting for $9.87 \%$ of all malignant tumor cases $(6,7)$ and the mortality rate of colon cancer in 2015 was $\sim 8 \%$ (8). At present, the primary treatment for colon cancer is surgery, supplemented by chemoradiotherapy, gene therapy, targeted therapy and other modalities; however, the strong invasive and migratory ability of colon cancer lead to a poor prognosis and low survival rates (9). Therefore, it is of great significance to explore the factors and signaling pathways associated with colon cancer cell proliferation and invasion in order to improve the clinical treatment of colon cancer and patient prognosis.

Nuclear receptor subfamily 3, group C, member 2 (NR3C2) is a nuclear transcription factor that encodes the MR protein (also known as the halocorticoid receptor) (10). NR3C2 is downregulated in several types of cancer and has been demonstrated to serve a tumor suppressor role. In colon cancer, microRNA (miR)-4709 can promote the proliferation, migration and invasion of colon adenocarcinoma cells and can target the downregulation of NR3C2 (11), indirectly indicating that NR3C2 may exert a tumor suppressive effect. However, the mechanism of its role in colon cancer remains to be elucidated. NR3C2 encodes a corticosteroid receptor, which has been reported to promote angiogenesis in colon cancer (12). In addition, the AKT/ERK signaling pathway is an important signaling pathway affecting angiogenesis (13). Research by Yang et al (14) demonstrated that NR3C2 can negatively regulate the AKT signaling pathway in pancreatic cancer. Therefore, the aim of the present study was to investigate whether NR3C2 can inhibit the proliferation, migration, invasion and angiogenesis of colon cancer cells through inhibiting the AKT/ERK signaling pathway, although 
the present study is not the first to detect NR3C2 expression in colon cancer, it will provide a reference for understanding the mechanism of NR3C2 in colon cancer.

\section{Materials and methods}

Cell culture. Normal colonic mucosa cells (NCM460), colon cancer cell lines (LoVo, CaCo2, SW1116, SW480 and HCT-116) and immortalized cell line human umbilical vein endothelial cells (HUVECs) were obtained from The Cell Bank of Type Culture Collection of The Chinese Academy of Sciences. All cells were cultured in the DMEM supplemented with $10 \%$ FBS (both from Gibco; Thermo Fisher Scientific, Inc.) in a humidified incubator at $37^{\circ} \mathrm{C}$ with $5 \% \mathrm{CO}_{2}$.

Cell viability assay. LoVo cells were plated in a 96-well plate $\left(5 \times 10^{3}\right.$ cells/well) and then pre-incubated for $24 \mathrm{~h}$ in a humidified incubator at $37^{\circ} \mathrm{C}$ with $5 \% \mathrm{CO}_{2}$. A total of $10 \mu \mathrm{l}$ Cell Counting Kit-8 (CCK-8) solution (Beyotime Institute of Biotechnology; cat. no. C0037) was added to each well of the plate. Light absorbance at 450-nm was measured using a microplate reader (BioTek Instruments, Inc.).

Cell transfection. The NR3C2 overexpression vector, pcDNA3.1-NR3C2 (Oe-NR3C2), and empty control vector, pcDNA3.1-NC (Oe-NC), were synthesized by Shanghai GeneChem Co., Ltd. The cells were inoculated on 12-well plates at a density of $3 \times 10^{5}$ cells/well and cultured in a $5 \% \mathrm{CO}_{2}$ incubator at $37^{\circ} \mathrm{C}$ for $24 \mathrm{~h}$. Following incubation, cells were transfected with the aforementioned plasmids $(50 \mathrm{ng} / \mathrm{ml})$ using Lipofectamine ${ }^{\circledR} 2000$ (Invitrogen; Thermo Fisher Scientific, Inc.), according to the manufacturer's protocols. Following transfection for $48 \mathrm{~h}$ at $37^{\circ} \mathrm{C}$ in a $5 \% \mathrm{CO}_{2}$ humid incubator, the protein expression level was evaluated by reverse transcription-quantitative (RT-q) PCR.

Wound healing and Transwell assays. For the wound healing assays, transfected LoVo cells were cultured in 6-well plates to $70-80 \%$ confluency, after which the monolayer was scratched with a $200-\mu 1$ sterile pipette tip. After washing with PBS, the cells were cultured in serum-free medium and images were captured at 0 and $24 \mathrm{~h}$ under an optical microscope (magnification, $\mathrm{x} 100$ ).

For the Transwell assays, the invasive ability of cells was evaluated using Transwell chambers coated with Matrigel (BD Biosciences) as previously described (15). Briefly, LoVo cells $\left(3 \times 10^{4}\right.$ cells) were plated in the plasma-free medium. The upper chamber was pre-coated with Matrigel at $37^{\circ} \mathrm{C}$ for $30 \mathrm{~min}$ (Sigma-Aldrich; Merck KGaA). A total of $0.1 \mathrm{ml}$ cell suspension was added to the upper chamber and the lower chamber was filled with medium supplemented with $20 \%$ FBS. Cells were cultured for $24 \mathrm{~h}$. Subsequently, the upper chamber was collected and cleaned and the cells that had invaded were stained with $0.5 \%$ crystal violet (Sigma-Aldrich; Merck KGaA) at room temperature for $10 \mathrm{~min}$. The stained cells were counted under an optical microscope (magnification, x200).

Colony formation assay. The cells were inoculated in 6 -well plates at the density of $4 \times 10^{2}$ cells/well for 14 days.
Subsequently, the cells were fixed with $70 \%$ ethanol and then stained with $0.05 \%$ crystal violet for $20 \mathrm{~min}$ at $37^{\circ} \mathrm{C}$. The number of colonies formed ( $>50$ cells/colony) were counted by an Olympus BX40 light microscope (Olympus Corporation).

ELISA. An ELISA kit (Nanjing Jiancheng Bioengineering Institute; cat. no. H044-1) was used to analyze the levels of VEGF according to the manufacturer's protocol. Each group was quantified using an Automatic Microplate Reader (Syngene).

HUVEC tube formation assay. Matrigel matrix glue, 24-well culture plates and pipette tips were placed at $4^{\circ} \mathrm{C}$ overnight. The culture medium of HUVECs in each group was changed to serum-free culture medium for $24 \mathrm{~h}$. Next, the cells were digested with $0.25 \%$ trypsin EDTA solution and were aspirated into a single-cell suspension following termination of digestion. After counting the number of cells, the cell density was adjusted to $7.5 \times 10^{6} \mathrm{ml}$. A total of $250 \mu 1$ Matrigel was added to each well of the 24 -well plate and the plate was placed in the incubator at $37^{\circ} \mathrm{C}$ with $5 \% \mathrm{CO}_{2}$ with saturated humidity and allowed to cure for $30 \mathrm{~min}$. The culture medium containing $300 \mu \mathrm{l}$ serum was mixed with $10 \mu \mathrm{l}$ single-cell suspension and then added to the 24-well plates. After culturing for $10 \mathrm{~h}$, five fields were randomly selected for observation under an inverted microscope (Olympus Corporation; magnification, $\mathrm{x} 40$ ).

$R T$ - $q P C R$. Total RNA from LoVo cells $\left(1 \times 10^{6}\right.$ cells/well) was extracted using TRIzol ${ }^{\circledR}$ reagent (Thermo Fisher Scientific, Inc.). Subsequently, according to the manufacturer's protocol. RNA concentration and quantification were assessed using a NanoDrop spectrophotometer (Thermo Fisher Scientific, Inc.). Following DNase I digestion, total RNA was reverse transcribed into cDNA using a QuantiTect Reverse Transcription kit (Applied Biosystems; Thermo Fisher Scientific, Inc.), according to the manufacturer's protocol. Subsequently, qPCR was performed using a QuantiTect SYBR Green PCR kit (Qiagen $\mathrm{GmbH}$ ), according to the manufacturer's protocol. The following thermocycling conditions were used for qPCR: $95^{\circ} \mathrm{C}$ for $10 \mathrm{~min}$; followed by 40 cycles of $95^{\circ} \mathrm{C}$ for $10 \mathrm{sec}$ and $60^{\circ} \mathrm{C}$ for $60 \mathrm{sec}$. The following primers (GenScript) were used for qPCR: NR3C2 forward, 5'-GATTGACAGTTGGTC GGC-3' and reverse, 5'-TTAGTCAGCTCAGGCTTGC-3' and GAPDH forward, 5'-AGCCACATCGCTCAGACAC-3' and reverse, 5'-GCCCAATACGACCAAATCC-3'. mRNA expression levels were quantified using the $2^{-\Delta \Delta \mathrm{Cq}}$ method (16) and normalized to the internal reference gene GAPDH. The experiments were performed in triplicate.

Total protein extraction and western blotting. Total protein was extracted from LoVo cells using radioimmunoprecipitation assay (RIPA) lysis buffer (Beyotime Institute of Biotechnology; cat. no. P0013C) and quantified using a Pierce BCA Protein assay kit (Thermo Fisher Scientific, Inc.). Following denaturing, electrophoresis was performed using SDS-PAGE with $12 \%$ SDS gels. Following protein transfer to PVDF membranes, the membranes were blocked in $5 \%$ fat-free milk for $2 \mathrm{~h}$ at room temperature. The membranes were incubated with primary antibodies (all purchased from Abcam) 
A

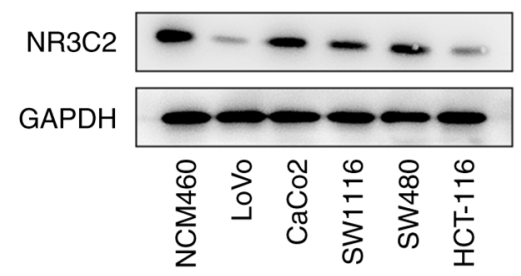

B

NR3C2

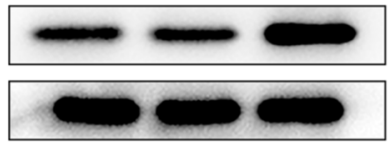

GAPDH

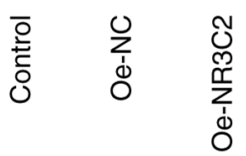
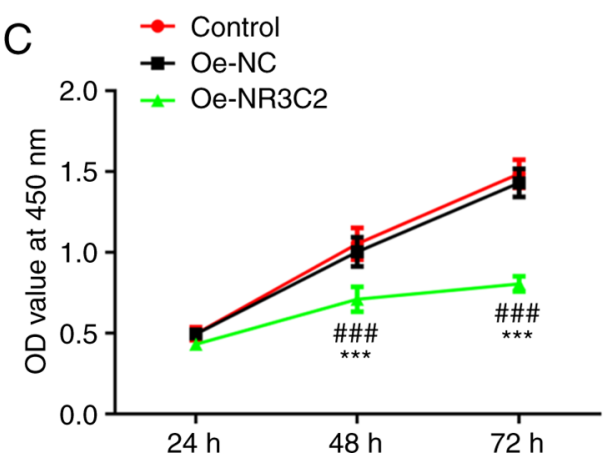
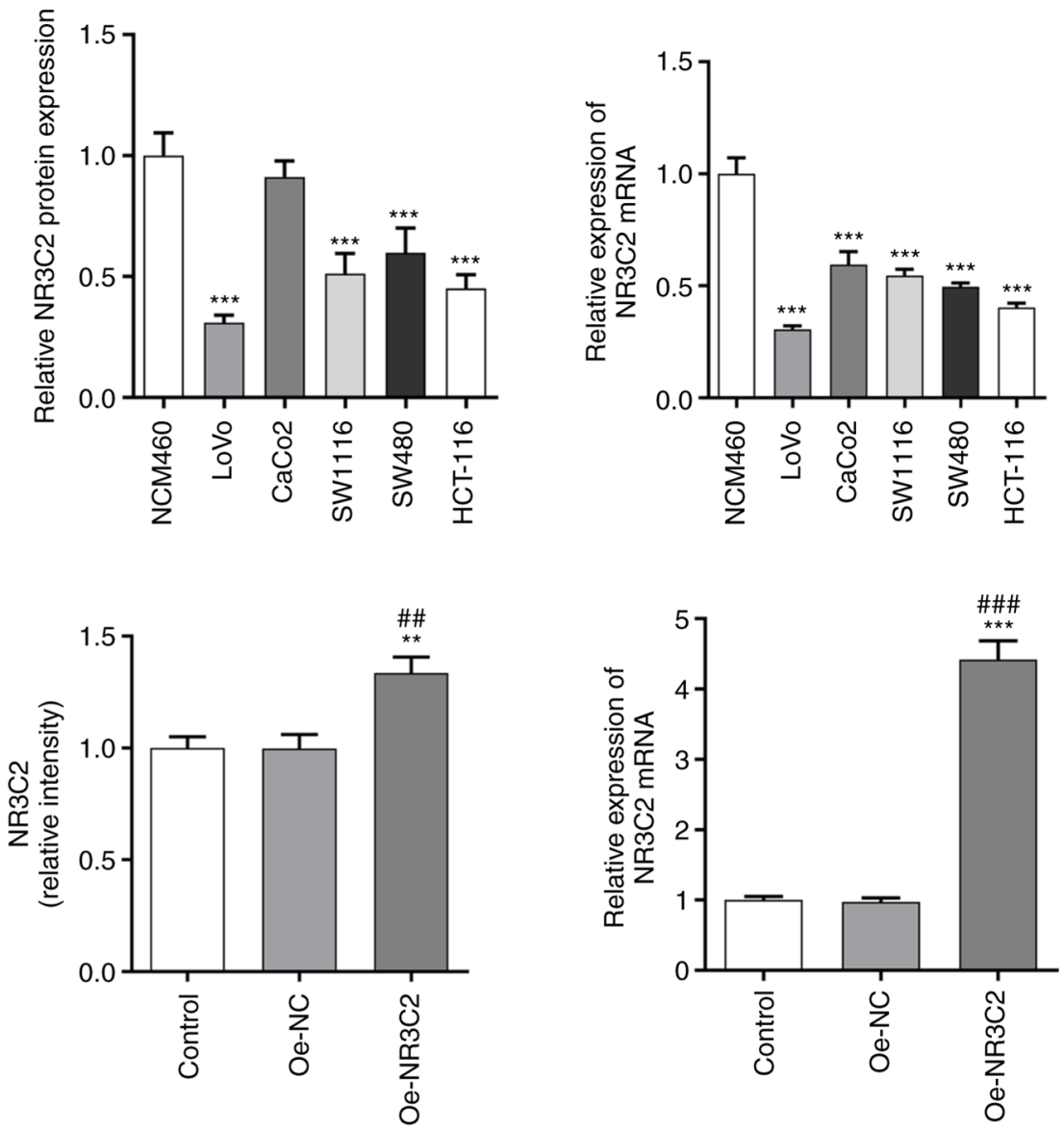

$\mathrm{D}$
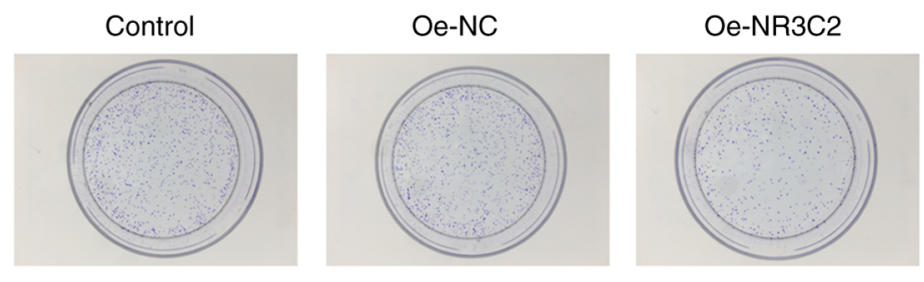

Figure 1. Overexpression of NR3C2 inhibited the proliferation of LoVo cells. (A) The expressions of NR3C2 in normal intestinal mucosa cells and colon cancer cell lines (CaCo2, SW1116, SW480, HCT-116 and LoVo) were detected by western blot and RT-qPCR. (B) Expression levels of NR3C2 in the LoVo were detected by western blot and RT-qPCR. (C) CCK-8 and (D) clone formation assays were performed to determine cell proliferation. ${ }^{* *} \mathrm{P}<0.01,{ }^{* * *} \mathrm{P}<0.001 \mathrm{vs}$. NCM460 or control; ${ }^{\# \#} \mathrm{P}<0.01,{ }^{\# \# \#} \mathrm{P}<0.001$ vs. Oe -NC. NR3C2, nuclear receptor subfamily 3, group C, member 2; RT-qPCR, reverse transcription-quantitative PCR; Oe, overexpression; NC, negative control.

against, NR3C2 (1:5,000; cat. no. ab64457), MMP2 (1:1,000; cat. no. ab92536), MMP9 (1:1,000; cat. no. ab76003), AKT (1,000; cat. no. ab18785), phosphorylated (p)-AKT (1:1,000; cat. no. ab38449), VEGFR2 (1:5,000; cat. no. ab134191), p-VEGFR2 (1:1,000; cat. no. ab5473), ERK $(1: 1,000$; cat. no. ab32537), p-ERK (1,000; cat. no. ab194776) and GAPDH $\left(1: 10,000\right.$; cat. no. ab181602) overnight at $4^{\circ} \mathrm{C}$. This was followed by incubation with IgG-horseradish peroxidase-conjugated goat anti-rabbit secondary antibody $(1: 3,000$; cat. no. ab6721) for $1 \mathrm{~h}$ at room temperature. Signals were visualized using ECL reagent (MilliporeSigma). Densitometry analysis was performed using ImageJ software (version 1.46; National Institutes of Health) with GAPDH as the loading control.
Statistical analysis. All experiments were repeated at least three times independently and results are expressed as the mean \pm standard deviation. Statistical analyses were performed using SPSS version 19.0 (IBM Corp.). One-way ANOVA followed by Tukey's post hoc test was used to compare differences between groups. $\mathrm{P}<0.05$ was considered to indicate a statistically significant difference.

\section{Results}

NR3C2 expression is downregulated in colon cancer cells. The expression levels of NR3C2 in normal intestinal mucosa cells and colon cancer cell lines $(\mathrm{CaCo} 2, \mathrm{SW} 1116$, SW480, HCT-116 and LoVo) was detected by western blotting and 

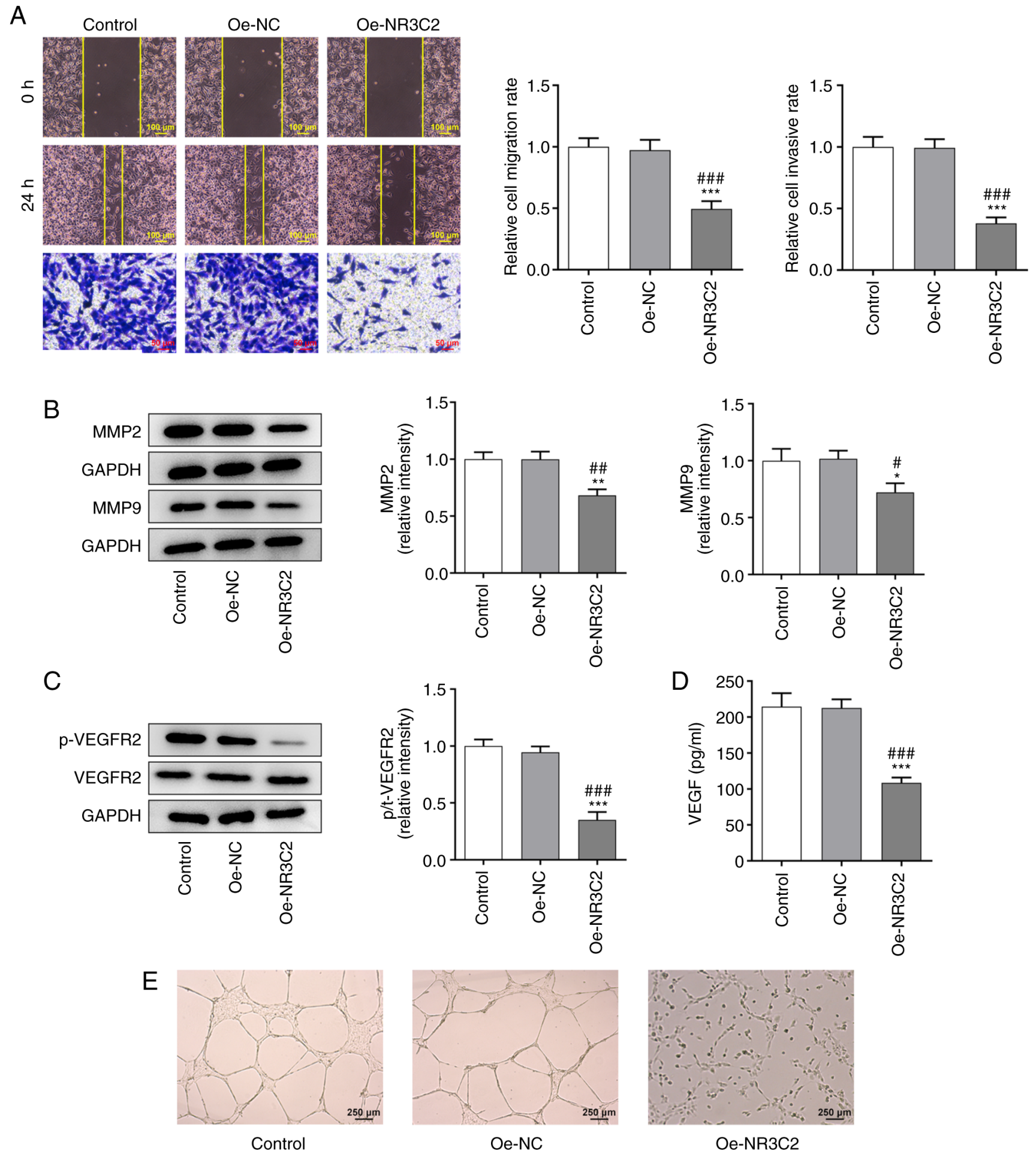

Figure 2. Overexpression of NR3C2 inhibitions migration, invasion and angiogenesis of LoVo cells. (A) Wound-healing and Transwell assays were used to detect cell migration and invasion. (B) Western blotting was used to detect the expression of invasion-related proteins MMP2 and MMP9. (C) Western blotting was used to detect the expression of invasion-related proteins p-VEGFR2 and VEGFR2. (D) VEGF levels were assessed using ELISA. (E) Tubule formation experiment was used to detect the angiogenesis of LoVo cells (magnification, $\mathrm{x} 40$ ). ${ }^{*} \mathrm{P}<0.05,{ }^{* * *} \mathrm{P}<0.01,{ }^{* * * *} \mathrm{P}<0.001$ vs. control; ${ }^{\# P} \mathrm{P}<0.05$, ${ }^{\# \#} \mathrm{P}<0.01,{ }^{\# \# \#} \mathrm{P}<0.001$ vs. Oe-NC. NR3C2, nuclear receptor subfamily 3, group C, member 2; p-, phosphorylated; Oe, overexpression; NC, negative control.

RT-qPCR. As demonstrated in Fig. 1A, NR3C2 expression was downregulated in a variety of colon cancer cells $(\mathrm{CaCo} 2$, SW1116, SW480, HCT-116 and LoVo) and the expression of NR3C2 was lowest in LoVo cells. Therefore, it was hypothesized that LoVo cells are representative to study the role of $\mathrm{NR} 3 \mathrm{C} 2$ in colon cancer and the LoVo cell line was selected for all subsequent experiments, which will be conducive to the significance of experimental results $(11,14)$.

Overexpression of NR3C2 inhibits the proliferation of LoVo cells. Next, the expression of NR3C2 was increased by transfection of an Oe-NR3C2 vector into LoVo cells. Compared with the 
A
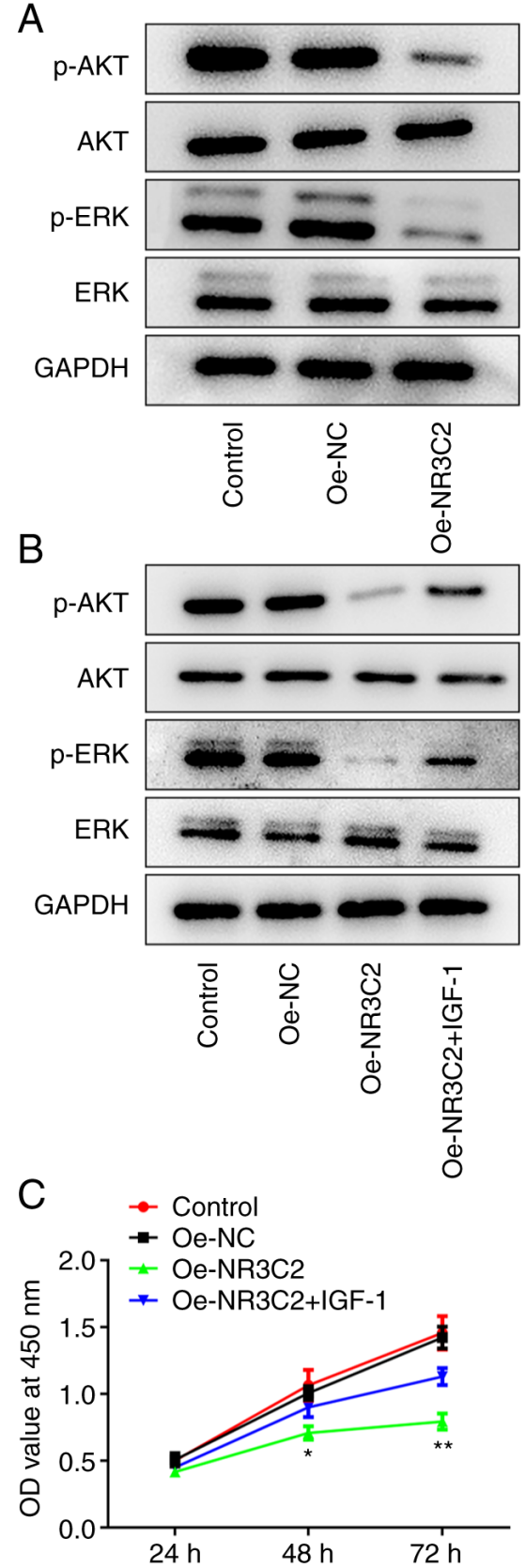
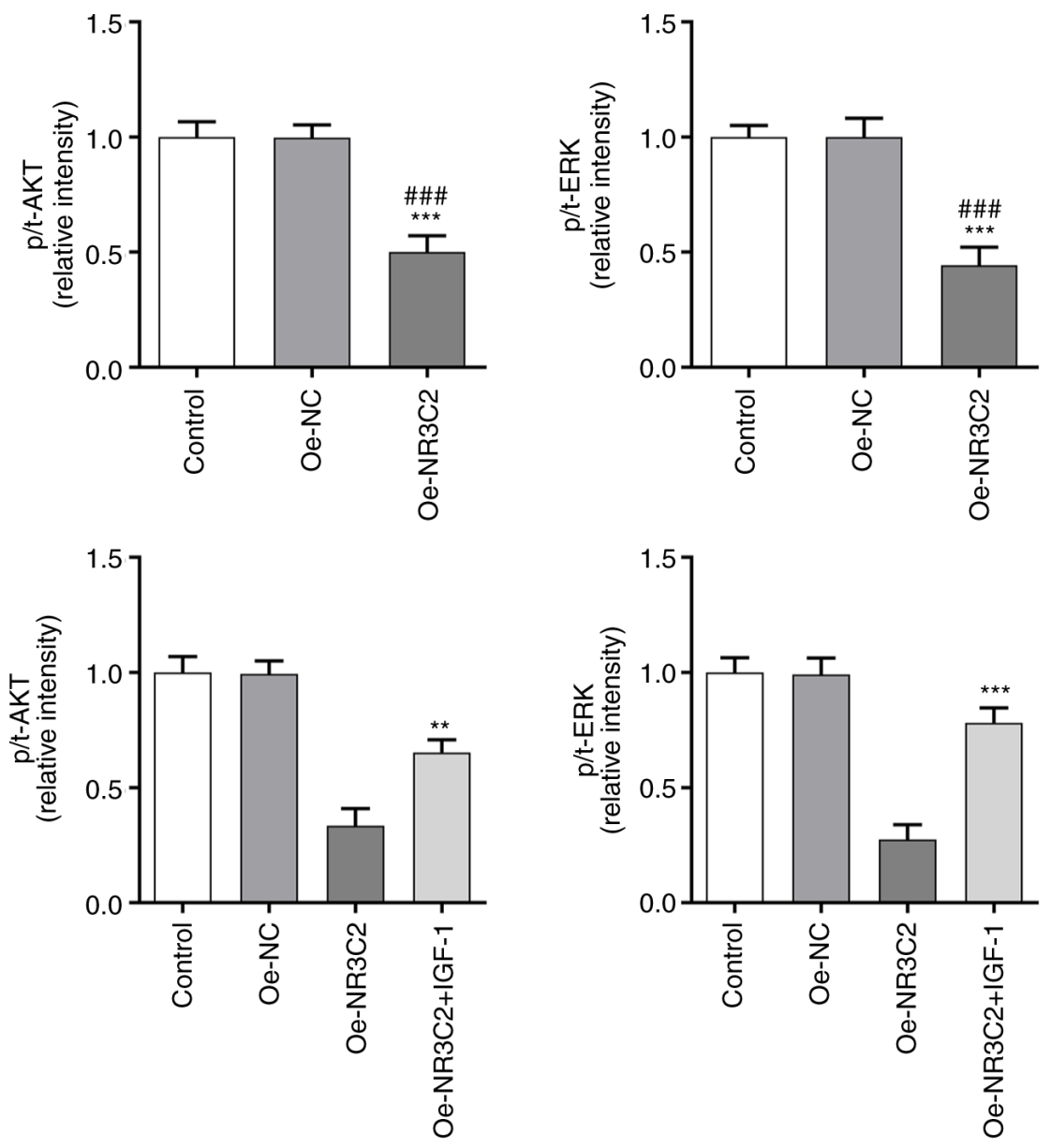

$\mathrm{D}$

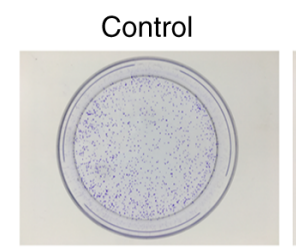

Oe-NC

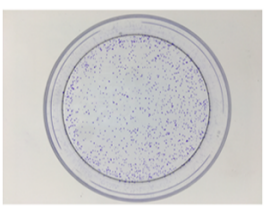

Oe-NR3C2

Oe-NR3C2+IGF-1

Figure 3. NR3C2 inhibits the proliferation and cloning of colon cancer cells through the AKT/ERK signaling pathway. (A) The expressions of p-AKT, AKT, ERK and p-ERK in LoVo cells were detected by western blotting. (B) The expressions of p-AKT, AKT, ERK and p-ERK in LoVo cells were detected by western blotting. (C) CCK-8 and assays (D) clone formation were performed to determine the cell proliferation. ${ }^{*} \mathrm{P}<0.05,{ }^{* * *} \mathrm{P}<0.01,{ }^{* * *} \mathrm{P}<0.001$ vs. Oe-NR3C2 or control; \#\#" $\mathrm{P}<0.001$ vs. Oe-NC. NR3C2, nuclear receptor subfamily 3, group C, member 2; p-, phosphorylated; Oe, overexpression; NC, negative control; IGF, insulin growth factor-1.

Oe-NC group, the expression of NR3C2 in the Oe-NR3C2 group was significantly higher (Fig. 1B). CCK-8 and colony formation assays were performed to assess cell proliferation. As demonstrated in Fig. 1C and D, overexpression of NR3C2 significantly reduced cell viability compared with the Oe-NC group.

Overexpression of NR3C2 inhibits migration and invasion of LoVo cells. Wound healing and Transwell assays were used to assess cell migration and invasion. As demonstrated in Fig. 2A, overexpression of NR3C2 significantly reduced the migration and invasion of LoVo cells. Western blotting was used to detect the expression of the invasion-related proteins, MMP2 and MMP9. As demonstrated in Fig. 2B, overexpression of NR3C2 significantly decreased the expression of MMP2 and MMP9 compared with the Oe-NC group.

Overexpression of NR3C2 inhibits angiogenesis in LoVo cells. The expression levels of angiogenesis-related proteins were detected by western blotting and the results demonstrated that the expression of phosphorylated- (p-)VEGF receptor 2 was significantly decreased in the Oe-NR3C2 group (Fig. 2C). VEGF levels detected by ELISA kit demonstrated that overexpression of $\mathrm{NR} 3 \mathrm{C} 2$ inhibited the 

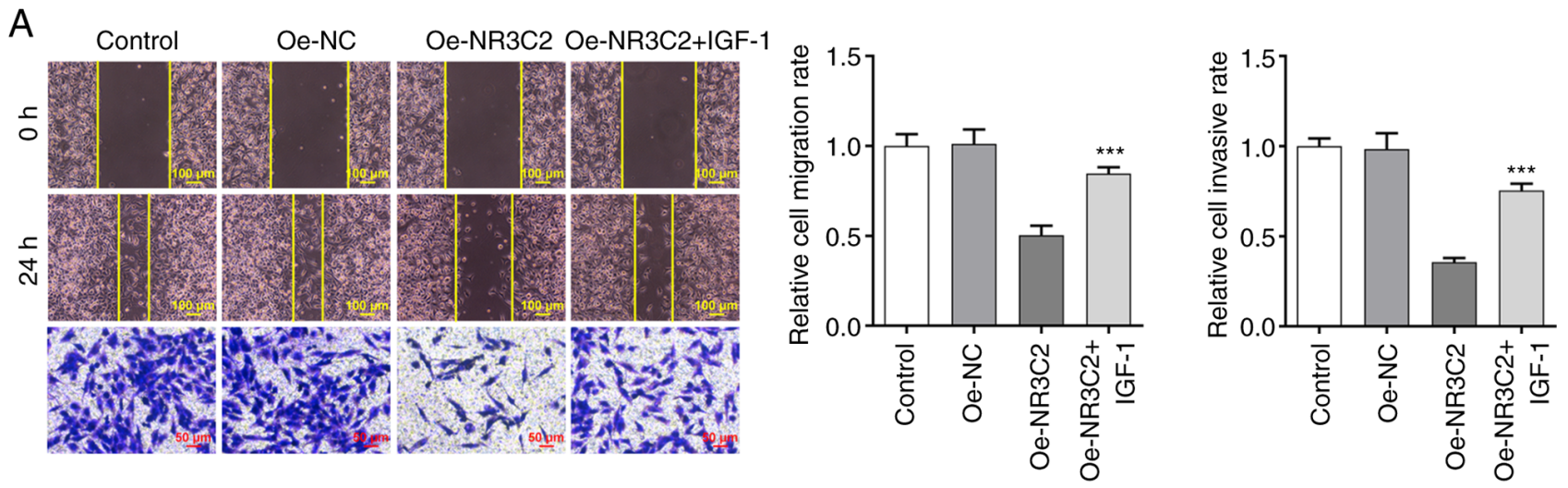

B
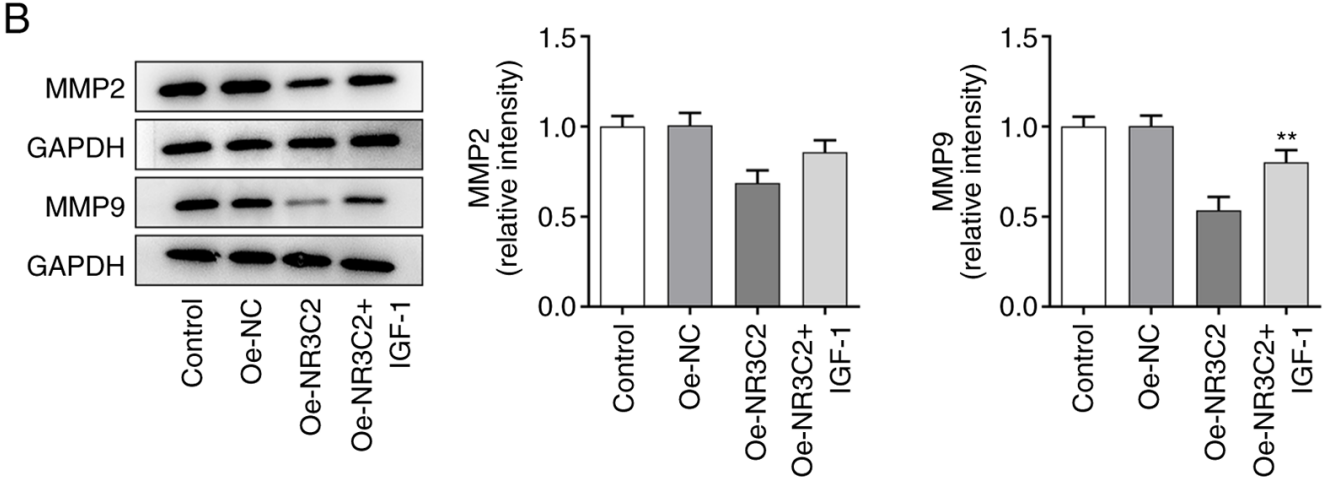

E
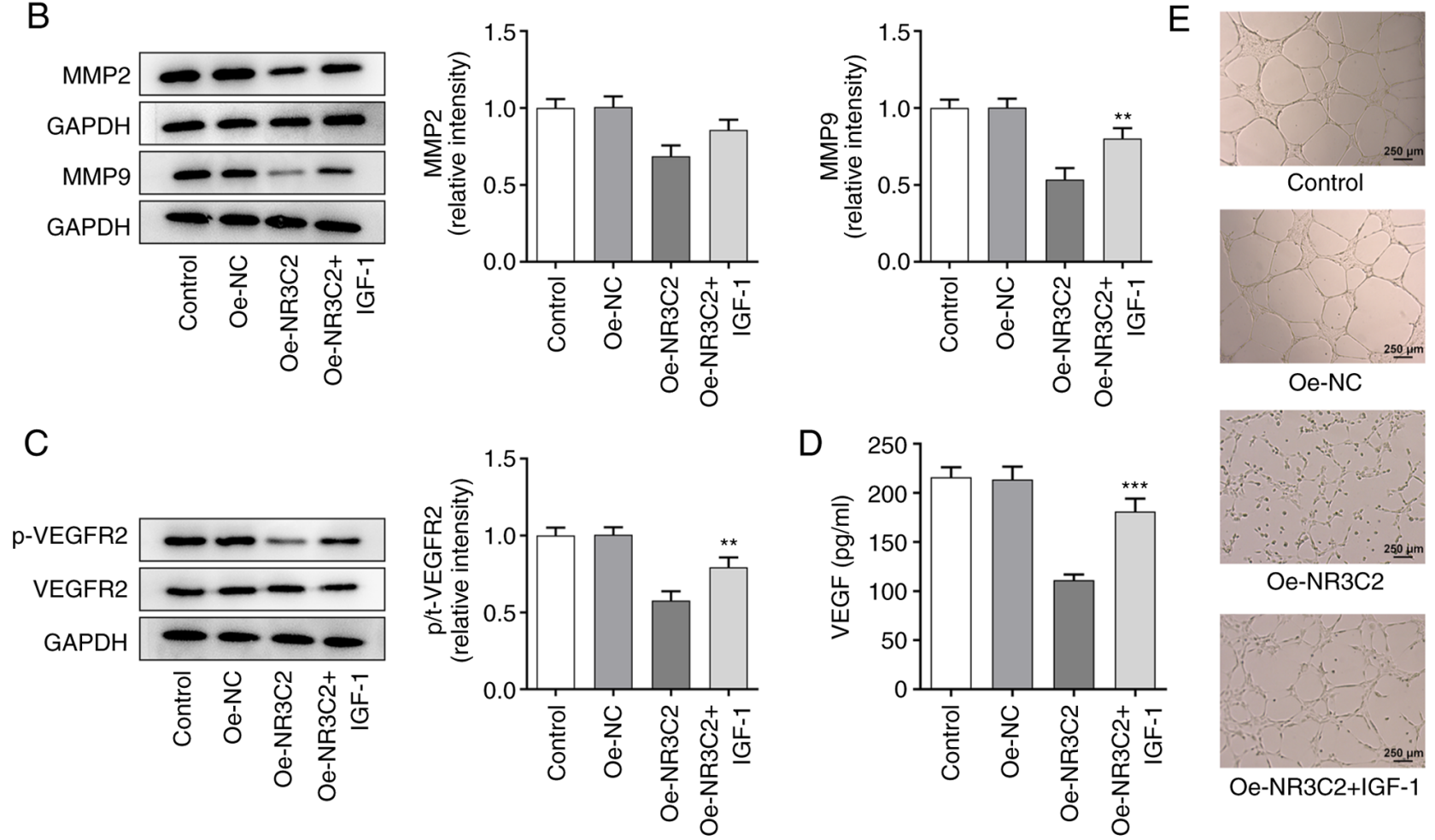

Oe-NR3C2+IGF-1

Figure 4. NR3C2 inhibits the migration, invasion and of colon cancer cells through the AKT/ERK signaling pathway. (A) Wound-healing and Transwell assays were used to detect cell migration and invasion. (B) Western blotting was used to detect the expression of invasion-related proteins MMP2 and MMP9. (C) Western blotting was used to detect the expression of invasion-related proteins p-VEGFR2 and VEGFR2. (D) VEGF levels were assessed using ELISA. (E) Tubule formation experiment was used to detect the angiogenesis of LoVo cells. ${ }^{* * *} \mathrm{P}<0.01,{ }^{* * * *} \mathrm{P}<0.001$ vs. Oe-NR3C2. NR3C2, nuclear receptor subfamily 3 , group C, member 2; p-, phosphorylated; Oe, overexpression; NC, negative control; IGF, insulin growth factor-1.

expression of VEGF (Fig. 2D). The results of the tube formation assays further demonstrated that NR3C2 overexpression inhibited angiogenesis in colon cancer cells (Fig. 2E).

Overexpression of NR3C2 inhibits the AKT/ERK signaling pathway in colon cancer cells. Yang et al (14) demonstrated that NR3C2 can negatively regulate the AKT signaling pathway; thus, western blotting was used to detect the expression levels of proteins associated with the AKT/ERK signaling pathway. Compared with the Oe-NC group, the overexpression of NR3C2 significantly reduced the expression of p-AKT and p-ERK (Fig. 3A).

NR3C2 inhibits the proliferation and colony formation of colon cancer cells through the AKT/ERK signaling pathway. To further investigate the role of NR3C2 in colon cancer, AKT agonist insulin growth factor-1 (IGF-1; $10 \mathrm{nM}$ ) was added to the medium. Western blotting demonstrated that overexpression of NR3C2 inhibited the expression of p-AKT and p-ERK and this was reversed by IGF-1 (Fig. 3B). CCK-8 and colony formation assays were used to assess cell proliferation and the results demonstrated that both were reduced by NR3C2 overexpression and increased by IGF-1 treatment (Fig. 3C-D).

NR3C2 inhibits the migration and invasion of colon cancer cells via the AKT/ERK signaling pathway. Transwell and wound healing assays were used to detect cell invasion and migration and it was found that NR3C2 overexpression could inhibit the metastasis of LoVo cells, whereas IGF-1 treatment enhanced invasion and migration (Fig. 4A). AKT is located upstream of ERK, so AKT/ERK can act as a cascade reaction to regulate the expression of MMPs (17), as demonstrated 
in Fig. 4B, NR3C2 overexpression inhibited the expression of metastasis-related proteins MMP2 and MMP9 in LoVo cells and IGF-1 treatment increased the expression of MMP2 and MMP9 (Fig. 4B). As demonstrated in Fig. 4C, IGF-1 markedly increased the expressions of $\mathrm{p}$-VEGFR2 compared with the Oe-NR3C2 group by western blot. in addition, VEGF levels detected by ELISA kit demonstrated that overexpression of NR3C2 inhibited the expression of VEGF, which reversed by IGF-1 (Fig. 4D). Tubule formation test was used to detect angiogenesis. Compared with the control group, tubule formation rate of HUVEC in Oe-NR3C2 group was significantly decreased and tubule formation rate was significantly increased after IGF-1 was added (Fig. 4E). Taken together, NR3C2 inhibits the migration, invasion and of colon cancer cells through the AKT/ERK signaling pathway.

\section{Discussion}

The present study demonstrated that NR3C2 expression was downregulated in colon cancer cells. It has been demonstrated that the expression of $\mathrm{NR} 3 \mathrm{C} 2$ is higher in normal tissues compared with cancerous tissues (14). Patients with CRC with upregulated expression of $\mathrm{NR} 3 \mathrm{C} 2$ have a longer 5-year overall survival (OS) rate, earlier-stage disease and lower rate of lymphatic and distant metastasis (11). Colon cancer is more common than rectal cancer and, consistent with the findings of the present study, the 5-year OS rate of colon cancer has been found to be higher than that of rectal cancer $(6,18)$. Several studies have demonstrated that $\mathrm{NR} 3 \mathrm{C} 2$ is a tumor suppressor gene and NR3C2 expression of the MR protein can inhibit angiogenesis and, thus, inhibit the progression of colorectal tumors (12). In other tumors, such as pancreatic cancer, NR3C2 also acts as a tumor suppressor gene, which can inhibit the proliferation and EMT of pancreatic cancer cells and increase sensitivity to certain chemotherapeutic agents $(14,19)$. In hepatocellular carcinoma, $\mathrm{NR} 3 \mathrm{C} 2$ can regulate the $\beta$-catenin signaling pathway to reduce the proliferation- and invasion-promoting effects of miR-766 on hepatocellular carcinoma cells (20). In renal cancer, the overexpression of NR3C2 is demonstrated to inhibit the proliferation, migration and other activities of renal cancer cells (21). Thus, it may be hypothesized that NR3C 2 may act as a tumor suppressor gene in CRC and inhibit the occurrence and development of CRC to some extent. The present study also confirmed that overexpression of NR3C2 inhibited proliferation, colony formation, migration and invasion of colon cancer cells. Overexpression of NR3C2 inhibited angiogenesis in colon cancer cells.

During tumor progression, pathological vascular growth is in a state of activation, which promotes the distal metastasis of tumor cells (22). VEGFA can effectively promote cell division and proliferation of tumor cells during tumor growth, invasion and metastasis (23). Clinical studies have demonstrated that the expression levels of VEGFA in the serum of patients with CRC is closely associated with the state of angiogenesis and is an independent predictor of prognosis $(23,24)$. The MAPK/ERK and PI3K/AKT signaling pathways serve important roles in vascular endothelial cell growth and angiogenesis of tumor cells (25). Studies have demonstrated that decreased VEGFA expression can inhibit the activation of the MAPK/ERK and PI3K/AKT signaling pathways, thus serving an anti-angiogenic role $(26,27)$. Angiogenesis is important for tumor growth and metastasis. Studies have demonstrated that the expression levels of VEGF in the tissues of patients with cancer is significantly higher compared with that in normal tissues (28). VEGF promotes angiogenesis by activating proliferation and invasion of vascular endothelial cells through AKT/PI3K/MAPK signaling (29). In the present study, overexpression of NR3C2 significantly reduced the expression levels of migration and invasion related proteins MMP2 and MMP9, which might be due to the fact that AKT was located upstream of ERK and AKT/ERK could regulate the expression of MMPs as a cascade reaction (17). Therefore, NR3C2 overexpression might inhibit the malignant process of colon cancer cells through AKT/ERK signaling pathway, which provided a reference for studying the mechanism of NR3C2 in colon cancer.

Although the present study obtained some meaningful results, there were some shortcomings, which could serve as the direction of future research. First, the expression level of NR3C2 was only detected in colon cancer cells, while the expression level in colon cancer tissues needs further investigation. Second, another colon cancer cell with low NR3C2 expression should be selected to further confirm the results of the present study. Third, it is necessary to fully discuss the relationship between NR3C2 and AKT/ERK signaling pathway through another agonist. Fourth, the results of the present study demonstrated that NR3C2 was downregulated in colon cancer cells, but the reason for its differential expression in different types of colon cancer cells needs further investigation, Finally, it should be emphasized that in future research it will be worthwhile to further detect cell activation and proliferation under NR3C2 inhibition on the basis of NR3C2 overexpression colon cancer cell.

In conclusion, the present study demonstrated that NR3C2 expression was downregulated in colon cancer tissues and that overexpression of NR3C2 inhibited the proliferation, migration, invasion and angiogenesis of colon cancer cells. In addition, NR3C2 affected the proliferation, migration, invasion and angiogenesis of colon cancer cells through the AKT/ERK signaling pathway. These findings may highlight novel targets for the treatment of colon cancer.

\section{Acknowledgements}

Not applicable.

\section{Funding}

This work was supported by the Natural Science Foundation of Jiangxi province (grant no. 20202ACB216003).

\section{Availability of data and materials}

All data generated or analyzed during this study are included in this published article.

\section{Authors' contributions}

JL conceptualized and designed the current study. JL and ZX acquired, analyzed and interpreted data. JL and ZX drafted the 
manuscript and revised it critically for important intellectual content. JL and ZX confirm the authenticity of all the raw data. Both authors agreed to be held accountable for the current study in ensuring questions related to the integrity of any part of the work are appropriately investigated and resolved. Both authors read and approved the final manuscript.

\section{Ethics approval and consent to participate}

Not applicable.

\section{Patient consent for publication}

Not applicable.

\section{Competing interests}

The authors declare that they have no competing interests.

\section{References}

1. Liu J, Yi J, Zhang Z, Cao D, Li L and Yao Y: Deoxyribonuclease 1-like 3 may be a potential prognostic biomarker associated with immune infiltration in colon cancer. Aging (Albany NY) 13: 16513-16526, 2021.

2. Troncone E, Marafini I, Stolfi C and Monteleone G: Involvement of Smad7 in inflammatory diseases of the gut and colon cancer. Int J Mol Sci 22: 3922, 2021.

3. Finotti M, Vitale A, Gringeri E, D'Amico FE, Boetto R, Bertacco A, Lonardi S, Bergamo F, Feltracco P and Cillo U: Colon rectal liver metastases: The role of the liver transplantation in the Era of the transplant oncology and precision medicine. Front Surg 8: 693387, 2021.

4. Tauriello DVF, Palomo-Ponce S, Stork D, Berenguer-Llergo A, Badia-Ramentol J, Iglesias M, Sevillano M, Ibiza S, Cañellas A, Hernando-Momblona X, et al: TGF $\beta$ drives immune evasion in genetically reconstituted colon cancer metastasis. Nature 554: 538-543, 2018.

5. Araghi M, Arnold M, Rutherford MJ, Guren MG, Cabasag CJ, Bardot A, Ferlay J, Tervonen H, Shack L, Woods RR, et al: Colon and rectal cancer survival in seven high-income countries 2010-2014: Variation by age and stage at diagnosis (the ICBP SURVMARK-2 project). Gut 70: 114-126, 2021.

6. Qin Q, Yang L, Sun YK, Ying JM, Song Y, Zhang W, Wang JW and Zhou AP: Comparison of 627 patients with right- and left-sided colon cancer in China: Differences in clinicopathology, recurrence, and survival. Chronic Dis Transl Med 3: 51-59, 2017.

7. Gao J, Yang M, Liu L, Guo S, Li Y and Cheng C: The aggressive surgical treatment and outcome of a colon cancer patient with COVID-19 in Wuhan, China. BMC Gastroenterol 20: 269, 2020.

8. Cao M, Li H, Sun D and Chen W: Cancer burden of major cancers in China: A need for sustainable actions. Cancer Commun (Lond) 40: 205-210, 2020.

9. Villalba M, Evans SR, Vidal-Vanaclocha F and Calvo A: Role of TGF- $\beta$ in metastatic colon cancer: It is finally time for targeted therapy. Cell Tissue Res 370: 29-39, 2017.

10. de Kloet ER, Van Acker SA, Sibug RM, Oitzl MS, Meijer OC, Rahmouni K and de Jong W: Brain mineralocorticoid receptors and centrally regulated functions. Kidney Int 57: 1329-1336, 2000.

11. Yu M, Yu HL, Li QH, Zhang L and Chen YX: miR-4709 overexpression facilitates cancer proliferation and invasion via downregulating NR3C2 and is an unfavorable prognosis factor in colon adenocarcinoma. J Biochem Mol Toxicol 33: e22411, 2019.

12. Tiberio L, Nascimbeni R, Villanacci V, Casella C, Fra A, Vezzoli V, Furlan L, Meyer G, Parrinello G, Baroni MD, et al: The decrease of mineralcorticoid receptor drives angiogenic pathways in colorectal cancer. PLoS One 8: e59410, 2013
13. Li X, Wang X, Li Z,Zhang Z and Zhang Y: Chemokine receptor 7 targets the vascular endothelial growth factor via the AKT/ERK pathway to regulate angiogenesis in colon cancer. Cancer Med 8: 5327-5340, 2019

14. Yang S, He P, Wang J, Schetter A, Tang W, Funamizu N, Yanaga K, Uwagawa T, Satoskar AR, Gaedcke J, et al: A novel MIF signaling pathway drives the malignant character of pancreatic cancer by targeting NR3C2. Cancer Res 76: 3838-3850, 2016.

15. Li W, Wu X and She W: lncRNA POU3F3 promotes cancer cell migration and invasion in nasopharyngeal carcinoma by up-regulating TGF- $\beta 1$. Biosci Rep 39: BSR20181632, 2019.

16. Livak KJ and Schmittgen TD: Analysis of relative gene expression data using real-time quantitative PCR and the 2(-Delta C(T)) method. Methods 25: 402-408, 2001.

17. Agraval H and Yadav UCS: MMP-2 and MMP-9 mediate cigarette smoke extract-induced epithelial-mesenchymal transition in airway epithelial cells via EGFR/Akt/GSK $3 \beta / \beta$-catenin pathway: Amelioration by fisetin. Chem Biol Interact 314: 108846, 2019.

18. Li X, Han F, Liu W and Shi X: PTBP1 promotes tumorigenesis by regulating apoptosis and cell cycle in colon cancer. Bull Cancer 105: 1193-1201, 2018.

19. Zhang Z, Che X, Yang N, Bai Z, Wu Y, Zhao L and Pei H: miR-135b-5p Promotes migration, invasion and EMT of pancreatic cancer cells by targeting NR3C2. Biomed Pharmacother 96: 1341-1348, 2017.

20. Yang C, Ma X, Guan G, Liu H, Yang Y, Niu Q, Wu Z, Jiang Y, Bian C, Zang Y and Zhuang L: MicroRNA-766 promotes cancer progression by targeting NR3C2 in hepatocellular carcinoma. FASEB J 33: 1456-1467, 2019.

21. Zhao Z, Zhang M, Duan X, Deng T, Qiu H and Zeng G: Low NR3C2 levels correlate with aggressive features and poor prognosis in non-distant metastatic clear-cell renal cell carcinoma. J Cell Physiol 233: 6825-6838, 2018.

22. Muche A, Bigl M, Arendt T and Schliebs R: Expression of vascular endothelial growth factor (VEGF) mRNA, VEGF receptor 2 (Flk-1) mRNA, and of VEGF co-receptor neuropilin (Nrp)-1 mRNA in brain tissue of aging Tg2576 mice by in situ hybridization. Int J Dev Neurosci 43: 25-34, 2015.

23. Mizuno R, Kawada K, Itatani Y, Ogawa R, Kiyasu Y and Sakai Y: The role of tumor-associated neutrophils in colorectal cancer. Int J Mol Sci 20: 529, 2019.

24. Boeckx N, Janssens K, Van Camp G, Rasschaert M Papadimitriou K, Peeters $M$ and Op de Beeck K: The predictive value of primary tumor location in patients with metastatic colorectal cancer: A systematic review. Crit Rev Oncol Hematol 121: 1-10, 2018.

25. Cheng JQ, Ruggeri B, Klein WM, Sonoda G, Altomare DA, Watson DK and Testa JR: Amplification of AKT2 in human pancreatic cells and inhibition of AKT2 expression and tumorigenicity by antisense RNA. Proc Natl Acad Sci USA 93: 3636-3641, 1996.

26. Cargnello M and Roux PP: Activation and function of the MAPKs and their substrates, the MAPK-activated protein kinases. Microbiol Mol Biol Rev 75: 50-83, 2011.

27. Gu Y, Wang Q, Guo K, Qin W, Liao W, Wang S, Ding Y and Lin J: TUSC3 promotes colorectal cancer progression and epithelial-mesenchymal transition (EMT) through WNT/ $\beta$-catenin and MAPK signalling. J Pathol 239: 60-71, 2016.

28. Chen H, Feng J, Zhang Y, Shen A, Chen Y, Lin J, Lin W, Sferra TJ and Peng J: Pien Tze Huang inhibits hypoxia-induced angiogenesis via HIF-1 $\alpha /$ VEGF-a pathway in colorectal cancer. Evid Based Complement Alternat Med 2015: 454279, 2015.

29. Wang R, Luo Z, Zhang H and Wang T: Tanshinone IIA reverses gefitinib-resistance in human non-small-cell lung cancer via regulation of VEGFR/Akt pathway. Onco Targets Ther 12: 9355-9365, 2019.

This work is licensed under a Creative Commons Attribution-NonCommercial-NoDerivatives 4.0 International (CC BY-NC-ND 4.0) License. 